\title{
Dynamics of unwinding of a simple entanglement
}

\author{
F W Wiegel† and J P J Michels $\ddagger$ \\ $\dagger$ Center for Theoretical Physics, Twente University, PO Box 217, 7500 AE Enschede, The \\ Netherlands \\ $\ddagger$ Van der Waals Laboratory, University of Amsterdam, PO Box 20216, 1000 HE Amsterdam, \\ The Netherlands
}

Received 3 February 1987

\begin{abstract}
The dynamics of unwinding of a simple entanglement is studied in two ways, firstly using an optimal path approximation in the Rouse model and secondly by simulating the movement of a more realistic model using Brownian molecular dynamics.
\end{abstract}

\section{Introduction}

The study of the dynamical behaviour of a system of entangled macromolecules leads to problems of staggering mathematical complexity. The few authors who have faced these problems have had to take refuge in extensive computer simulations which, indeed, provide numerical answers to some of the most urgent questions. For example, the dynamics of three-branched star molecules was studied numerically by Needs and Edwards (1983), also of Needs (1983), and other authors have simulated related many-polymer systems with either the Monte Carlo or the molecular dynamics method.

In this paper, we address a much simpler dynamical problem in which only a single polymer plays a role: a Gaussian chain is located in a plane and topologically entangled with a point of the plane. More generally, we consider a Gaussian chain in threedimensional space. At $t=0$, the chain is topologically entangled with some closed curve in space. The equilibrium statistical physics of the chain can be worked out analytically for a variety of geometries and chain-curve interactions (a recent review of these models, methods and solutions can be found in Wiegel (1983)). In this paper we propose to study the dynamics of this model in two ways: (i) analytically by combining the Rouse (1953) model of polymer dynamics with the topological features of the entanglement and (ii) numerically by simulating the motion by molecular dynamics. In this way, one hopes to gain some insight into the peculiar way in which the topology interferes with the ordinary relaxation of the chain.

The essential idea underlying the Rouse model is that, although the polymer chain as a whole can be out of equilibrium, any small part of it is so close to equilibrium that its elastic properties can be calculated from the equilibrium distribution. This leads to a model in which the polymer is represented by $N$ mass points, each of which has mass $m$, friction coefficient $\gamma$ and represents $n_{0}$ repeating units of mass $m_{0}$. Between these mass points, linear entropic forces act corresponding to the Gaussian statistics of the various chain segments.

Before applying the same idea to the dynamics of unwinding of a simple entanglement we summarise the results of the equilibrium theory for the basic case in which 
the chain is located in a plane, and where the obstacle is at the origin of coordinates (this case will be considered throughout the rest of this paper). One end of the chain is kept fixed in a position with polar coordinates $\left(r_{0}, \theta_{0}\right)$, where $0<r_{0}<\infty,-\pi<\theta_{0}<\pi$. Let the other end have polar coordinates $(r, \theta)$, where $\theta$ is increasing by $2 \pi$ for each counterclockwise turn around the origin, so $-\infty<\theta<+\infty$. If, for a particular configuration, $\theta$ is found to have a value in the interval $(2 n \pi-\pi, 2 n \pi+\pi)$ we shall say that the polymer has entanglement index $n$. It has been shown that the equilibrium configuration sum $Q(r, \theta)$ over all those polymer configurations which (i) start with $\left(r_{0}, \theta_{0}\right)$, (ii) end with $(r, \theta)$ and (iii) have entanglement index $n=0, \pm 1, \pm 2, \ldots$, is given by the integral

$Q(r, \theta)=\left(\pi N_{0} l^{2}\right)^{-1} \exp \left(-\frac{r_{0}^{2}+r^{2}}{N_{0} l^{2}}\right) \int_{-\infty}^{+\infty} I_{|k|}\left(\frac{2 r_{0} r}{N_{0} l^{2}}\right) \exp \left[\mathrm{i} k\left(\theta-\theta_{0}\right)\right] \mathrm{d} k$.

The number of repeating units is $N_{0}=N n_{0}$ and the length of a repeating unit is $l$ (see figure 1). The $I_{|k|}$ denote the modified Bessel functions. In $\S 2$ we adapt the Rouse model to the topology of this situation.

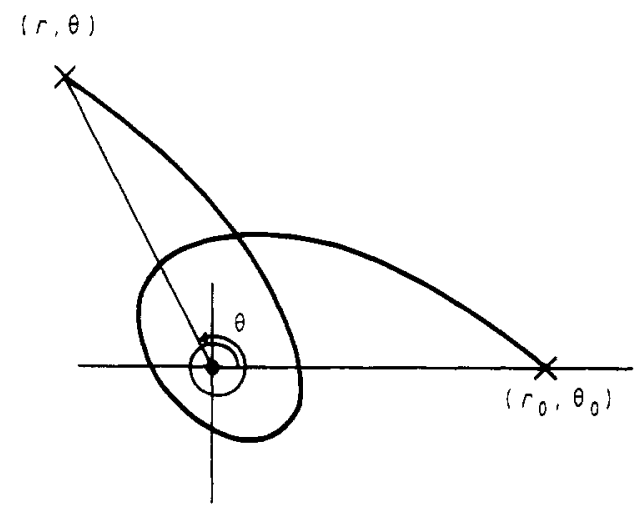

Figure 1. Basic geometry of the simple entanglement problem.

The analytical study which is going to form the subject of $\S 2$ cannot be pushed far enough to obtain explicit results. In view of this, we felt the need to supplement it with a numerical simulation. In $\$ 3$ we study the dynamics of unwinding with the Brownian molecular dynamics method which was used by us in a previous study of numerical hammagraphy (Michels and Wiegel 1986).

\section{Analytical considerations}

In the simplest model for the dynamics of unwinding one would separate completely the topological aspect from the frictional aspect. If the endpoint $\left(r_{0}, \theta_{0}\right)$ is kept fixed, the 'force' $\boldsymbol{F}(r, \theta)$ on the other endpoint is an entropic force equal to

$$
\boldsymbol{F}(r, \theta)=k_{\mathrm{B}} T \nabla \ln Q(r, \theta) \quad-\infty<\theta<+\infty .
$$

Here, $k_{\mathrm{B}}$ is Boltzmann's constant and $T$ is the absolute temperature. If one moves all mass and hydrodynamic friction to the endpoint of the chain, its dynamics can be 
described by a Langevin equation of the form

$$
M \frac{\mathrm{d}^{2} \boldsymbol{r}}{\mathrm{d} t^{2}}=\boldsymbol{F}-f \frac{\mathrm{d} \boldsymbol{r}}{\mathrm{d} t}+\boldsymbol{\varepsilon}
$$

where $M=N m$ is the total mass, $f$ is the total friction coefficient and $\varepsilon$ is a stochastic force simulating the heat motion of the solvent. Various comments are in order here.

(a) In the absence of hydrodynamic interactions between the repeating units, the friction coefficient is simply given by

$$
f=N \gamma .
$$

In the presence of hydrodynamic interactions one finds the empirical relation

$$
f \approx f_{0} N^{a}
$$

where $f_{0}$ is a constant with the same dimension as $f$ and where the exponent $a$ is in the region $0.5-0.6$, depending on the polymer-solvent interaction (Mijnlieff and Wiegel 1978).

(b) Note that the topological constraint is automatically taken into account when expression (2.1) is used for the entropic force: the fluctuations $\varepsilon$ can never sweep the chain across the obstacle at the origin because the "potential'

$$
V(r, \theta)=-k_{\mathrm{B}} T \ln Q(r, \theta) \quad-\infty<\theta<+\infty
$$

tends to $+\infty$ for $r \downarrow 0$ (this follows from (1.1) and the fact that the modified Bessel functions $I_{|k|}$ vanish for $r \downarrow 0$ ). Hence the dynamics which is implied by (2.1) and (2.2) has the desired property that the chain can only move around, and not through the obstacle at the origin.

(c) Just as in the Rouse theory one notes that the acceleration term in (2.2) is of the order $M r \tau^{-2}$, where $\tau$ is a typical relaxation time. As the friction term is of the order of $f r \tau^{-1}$, their ratio is of the order of $M f^{-1} \tau^{-1}$. As the typical relaxation time is of the order of $N^{2}$ this shows that (2.2) can be replaced by

$$
f \frac{\mathrm{d} \boldsymbol{r}}{\mathrm{d} t}=\boldsymbol{F}+\boldsymbol{\varepsilon}
$$

provided the polymer is very long.

It is of interest to study the consequences of (2.6) in the presence, as well as in the absence, of the fluctuation term $\varepsilon$. These two cases are the analogues in the present problem of treating a mechanical problem with quantum mechanics or classical mechanics. Moreover, they are also analogous to treating an optical problem with wave optics or with geometrical optics.

In the absence of the fluctuating term, (2.6) reduces to the integral equation

$$
\boldsymbol{r}(t)=\boldsymbol{r}(0)+\frac{1}{f} \int_{0}^{1} \boldsymbol{F}\left(\boldsymbol{r}\left(t^{\prime}\right)\right) \mathrm{d} t^{\prime} .
$$

Although the complicated form of $\boldsymbol{F}$ makes an analytical treatment very difficult, one can approximate the solution in various limiting cases.

(i) $r \gg l \sqrt{ } N_{0}$. In this case we can use the approximation

$$
Q(r, \theta)=(\text { constant }) \exp \left(-\frac{r^{2}}{N_{0} l^{2}}\right)
$$


from which the force is found using (2.1)

$$
F(r)=-\frac{2 k_{\mathrm{B}} T}{N_{0} l^{2}} r
$$

which is evidently independent of $\theta$. Substituting this into (2.6) with $\varepsilon=0$, one finds

$$
\frac{\mathrm{d} r}{\mathrm{~d} t}=-\frac{2 k_{\mathrm{B}} T}{N_{0} l^{2} f} r
$$

with solution

$$
r(t)=r(0) \exp \left(-\frac{2 k_{\mathrm{B}} T}{N_{0} l^{2} f} t\right)
$$

This initial part of the relaxation process consists of the polymer contracting radially towards the obstacle at $r=0$, with a relaxation time

$$
\tau_{1}=\frac{N_{0} l^{2} f}{2 k_{\mathrm{B}} T}
$$

Using (2.4) and the relation $N_{0}=N n_{0}$, one finds

$$
\tau_{1}=\frac{n_{0} l^{2} f_{0}}{2 k_{\mathrm{B}} T} N^{a+1}
$$

and hence an $N$ exponent in the region 1.5-1.6. In the Rouse model (2.3) one finds an $N$ exponent equal to 2 .

(ii) $\left(\theta-\theta_{0}\right) \gg 1$. Suppose the endpoint of the polymer has relaxed radially to the vicinity of the obstacle. This process has not changed the entanglement index, so for a highly entangled polymer one has $\left(\theta-\theta_{0}\right) \gg 1$. In this case, the integral (1.1) is dominated by the contributions from values of $|k| \ll 1$. Hence we can substitute the asymptotic formula for the modified Bessel functions

$$
I_{k}(z) \approx(z / 2)^{k} I_{0}(z) \quad|k| \ll 1
$$

(cf Abramowitz and Stegun 1965, e.g. 9.6.10) to find

$$
Q(r, \theta) \approx\left(\pi N_{0} l^{2}\right)^{-1} \exp \left(-\frac{r_{0}^{2}}{N_{0} l^{2}}\right) \frac{-2 \ln \left(r_{0} r / N_{0} l^{2}\right)}{\left[\ln \left(r_{0} r / N_{0} l^{2}\right)\right]^{2}+\left(\theta-\theta_{0}\right)^{2}} \quad\left|\theta-\theta_{0}\right| \gg 1
$$

This final stage of the relaxation process consists of the endpoint unwinding itself around the obstacle.

As we have assumed strong damping the endpoint will essentially move along the path of minimum potential energy. In order to find the shape of this path, we ask for which value of $r$ the potential is minimal, given a fixed value of $\theta-\theta_{0}$. In view of (2.5) the minimum of the potential $V$ corresponds to the maximum of $Q$. Solving the equation $(\partial Q / \partial r)_{\theta}=0$, one finds that the path followed by the endpoint is approximately described by a spiral of the form

$$
\frac{r}{l} \approx \frac{N_{0} l}{r_{0}} \exp \left[-\left(\theta-\theta_{0}\right)\right]
$$

It is easy to verify that the total length of this spiralling path is

$$
S=\int_{\theta_{0}}^{x}\left[r^{2}+\left(\frac{\mathrm{d} r}{\mathrm{~d} \theta}\right)^{2}\right]^{1 / 2} \mathrm{~d} \theta=N_{0} \sqrt{2} \frac{l^{2}}{r_{0}}
$$


The present part of the relaxation process is somewhat more complicated, but it is not difficult to show that the relaxation time is of the order of magnitude

$$
\tau_{2}=\frac{f}{k_{\mathrm{B}} T}\left(\frac{N_{0} l^{2}}{r_{0}}\right)^{2} .
$$

As $N_{0} \sim N, f \sim N^{a}$ and a typical value of $r_{0}$ is of the order $l \sqrt{ } N$, the exponent is seen to be $a+1$, and so again in the region 1.5-1.6. Comparing $\tau_{2}$ with $\tau_{1},(2.13)$, one finds that both processes play a role in the total relaxation process.

Collecting the results (2.7)-(2.18), which ignore the fluctuations, one finds that the endpoint of the polymer unwinds along a trajectory with a shape which is sketched in figure 2. We should stress that the actual movement of the endpoint could deviate substantially from the sketched trajectory as a result of the heat motion of the medium (the term $\varepsilon$ in (2.6)). An analytical calculation which takes these fluctuations into account is very difficult indeed. This justifies a numerical approach, which forms the subject of the next section.

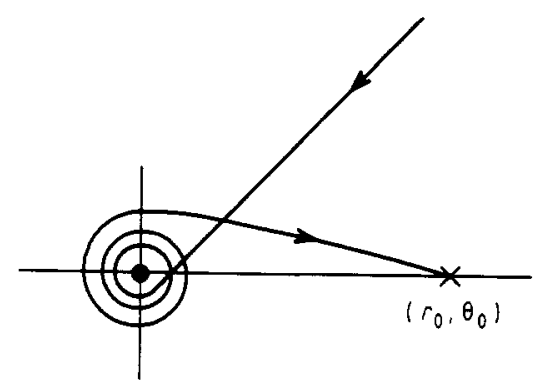

Figure 2. Quahtative feature of the 'average' trajectory followed by the endpoint of a disentangling polymer.

\section{Brownian molecular dynamics}

The model which forms the basis of the numerical simulation consists of a string of $N$ mass points constrained to move in a plane. Between each pair of neighbouring masses a spring force acts, which has a finite equilibrium distance $l$. The chain is shuffled around by random Brownian forces. All details are identical to the model previously used by Michels and Wiegel (1986) to which paper we refer for details. It should be pointed out that the proper way to generate (closed) polymer configurations has formed the subject of several recent papers. The various ways of applying the Monte Carlo method have been discussed by Frank-Kamenetskii and Vologodskii (1981). In the present study we use Brownian molecular dynamics. At the origin of coordinates one places an impenetrable disc of radius $l$. This is realised by defining a centrifugal force

$$
\begin{aligned}
\boldsymbol{F}\left(r_{i}\right) & =a \frac{\left(l-r_{i}\right)}{r_{i}} r_{i} & & \text { if } 0<r_{i}<l \\
& =0 & & \text { if } r_{i}>l .
\end{aligned}
$$

In this equation, the force constant $a$ is such that the force is strongly repulsive inside the disc and $r_{0}$ is the position of mass point $i=1,2, \ldots, N$. 
During the simulation we keep the location $r_{i}$ of the first mass point fixed in a position on the $x$ axis with polar coordinates $r_{1}, \theta_{1}=0$. At time $t=0$ the original positions $\boldsymbol{r}_{1}, \boldsymbol{r}_{2}, \ldots, \boldsymbol{r}_{N}$ are chosen to lie in some arbitrary way, such that the chain encircles the disc at the origin exactly once in a counterclockwise direction (i.e. $\left.\theta_{N}=2 \pi\right)$.

For $t>0$, we proceed in two steps. Firstly the movement of the chain is followed using the method of Michels and Wiegel (1986), with the extra restriction that the $N$ th mass point can only move along the positive $x$ axis $\left(\theta_{N}\right.$ fixed at $\left.2 \pi\right)$. After a certain length of time the initial configuration of the chain is randomised. Secondly, the restriction $\theta_{N}=2 \pi$ is dropped and all $N$ mass points $(i=1,2, \ldots, N)$ are permitted to move without constraints. During the ensuing process of unwinding, the polar coordinates $r_{N}(t), \theta_{N}(t)$ of the endpoint are stored in memory. This procedure was repeated many times (for several values of $N$ ) starting from a newly randomised initial configuration.

For each run the program calculated (i) the first passage time of the endpoint, i.e. the first time (after starting the second step) that $\theta_{N}(t)$ obtains a negative value and (ii) the values of $r_{N}(t)$ and $\theta_{N}(t)$ at narrowly spaced intermediate times. Afterwards, by averaging over the ensemble of many runs, we calculated (a) the averages $\left\langle r_{N}(t)\right\rangle$ and $\left\langle\theta_{N}(t)\right\rangle$ and (b) the distribution of the first passage time.

In our simulations we used chains with $N=64$ and $N=96$. For each value of $N$ we chose $r_{0} / l=7,9,12$ or 16 . For each combination of $N$ and $r_{0} / l$ we averaged over 500 runs. In figure 3 the behaviour of $\left\langle r_{N}(t)\right\rangle$ and $\left\langle\theta_{N}(t)\right\rangle$ is shown for the case $N=64$, $r_{0} / l=9$. It should be noticed that the limiting value of $\left\langle r_{N}(t)\right\rangle$ for large times is $\approx 11.5 l$, in excess of the value $9 l$ which one might have expected. This difference is due to the steric repulsion of the chain by the obstacle at the origin. It is advantageous for the chain (after the unwinding has been completed!) to assume configurations which are preferably located some distance away from the obstacle, hence this effect is purely entropic.

In figure 4 , we show the temporal behaviour of the function $p_{0}(t)$, which is defined as the probability that at time $t$ there has been at least one passage of the endpoint through $\theta_{N}=0$. A possible choice for the 'timescale for unwinding' would be the time $\tau_{0}$, where $p_{0}\left(\tau_{0}\right)=\frac{1}{2}$. The value of $\tau_{0}$ for the various values of $N$ and $r_{0} / l$ are

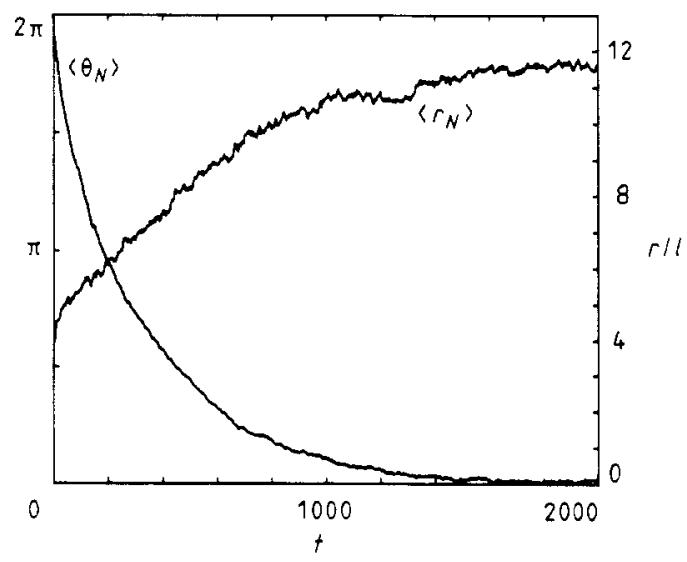

Figure 3. Average time behaviour of the radius $\left\langle r_{N}(t)\right\rangle$ and angle $\left\langle\theta_{N}(t)\right\rangle$ of a disentangling polymer. 


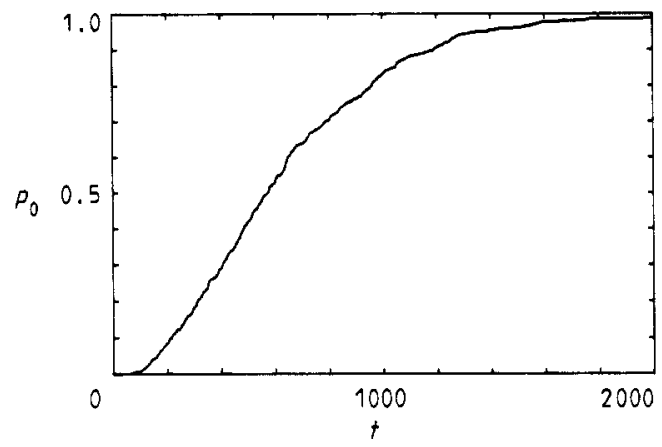

Figure 4. Time dependence of the function $p_{0}(t)$.

Table 1. The value of $\tau_{0}$ for different values of $r_{0} / l$ for chains with $N=64$ and $N=96$.

\begin{tabular}{rll}
\hline$r_{0} / l$ & $N=64$ & $N=96$ \\
\hline 7 & 640 & \\
9 & 560 & \\
12 & 330 & 840 \\
16 & 250 & 730 \\
\hline
\end{tabular}

listed in table 1. The unit of time in figures 3,4 and table 1 equals $l\left(m / k_{\mathrm{B}} T\right)^{1 / 2}$. It turned out that the qualitative behaviour of the functions in figures 3 and 4 is the same for all values of $N$ and $r_{0} / l$.

These simulations indicate the importance of the fluctuations, as has already been mentioned at the end of $\S 2$. The computing facilities needed were rather modest as all calculations were performed on a scalar mainframe. As the algorithm for Brownian molecular dynamics would be highly suitable for vectorisation, this form of numerical hammagraphy seems to carry some promise for the future.

\section{Acknowledgments}

The research reported in this paper was started during a stay of one of us (FWW) at the University of Cambridge. This author would like to thank Professor S F Edwards for his hospitality at the Cavendish Laboratory during the spring of 1985.

\section{References}

Abramowitz M and Stegun I A 1965 Handbook of Mathematical Functions (New York: Dover)

Frank-Kamenetskii M D and Vologodskii A V 1981 Usp. Fiz. Nauk 134 641-73 (Sov. Phys.-Usp. 24 679-96)

Michels J P J and Wiegel F W 1986 Proc. R. Soc. A 403 269-84

Mijnlief P F and Wiegel F W 1978 J. Polym. Sci. Polym. Phys. Ed. 16 245-63

Needs R J 1983 PhD Thesis University of Cambridge

Needs R J and Edwards S F 1983 Macromol. 16 1492-5

Rouse P E 1953 J. Chem. Phys. 21 1272-80

Wiegel F W 1983 Phase Transitions and Critical Phenomena vol 7, ed C Domb and J L Lebowitz (New York: Academic) pp 100-49 